\title{
Lexicographic Codes: Error-Correcting Codes from Game Theory
}

\author{
JOHN H. CONWAY AND N. J. A. SLOANE, FELlOW, IEEE
}

\begin{abstract}
Lexicographic codes, or lexicodes, are defined by various versions of the greedy algorithm. The theory of these codes is closely related to the theory of certain impartial games, which leads to a number of surprising properties. For example, lexicodes over an alphabet of size $B=2^{a}$ are closed under addition, while if $B=2^{2^{a}}$ the lexicodes are closed under multiplication by scalars, where addition and multiplication are in the nim sense explained in the text. Hamming codes and the binary Golay codes are lexicodes. Remarkably simple constructions are given for the Steiner systems $S(5,6,12)$ and $S(5,8,24)$. Several record-breaking constant weight codes are also constructed.
\end{abstract}

\section{INTRODUCTION}

$\mathrm{T}$ HIS PAPER is concerned with various classes of lexicographic codes, that is, codes that are defined by a greedy algorithm: each successive codeword is selected as the first word not prohibitively near (in some prescribed sense) to earlier codewords. For example, the very simplest class of lexicographic codes is defined as follows. We specify a base $B$ and a desired minimal Hamming distance $d$. The first codeword accepted is the zero word. Then we consider all base- $B$ vectors in turn, and accept a vector as a codeword if it is at Hamming distance at least $d$ from all previously accepted codewords. (An example with $B=3$ and $d=3$ can be seen in Table XI.)

One of our goals is to point out the essential identity between this kind of lexicographic coding theory and the theory of certain impartial games (see Section II). Then the Sprague Grundy theory of games has a number of interesting and surprising consequences for lexicographic codes (or lexicodes).

1) Unrestricted binary lexicodes are linear (Theorems $1,3)$.

2) For base $B=2^{a}$, unrestricted lexicodes are closed under nim-addition (Theorem 4).

3) For base $B=2^{2^{a}}$, unrestricted lexicodes are closed under nim-multiplication, which is an operation that converts the digits $\left\{0,1,2,3, \cdots, 2^{2^{a}}-1\right\}$ into a field (Theorem 5).

4) The constant weight binary lexicodes with minimal distance 4 have a rather subtle complete solution in terms of Welter's game (Section IV-C).

Manuscript received March 7, 1985; revised October 31, 1985

J. H. Conway is with the Department of Pure Mathematics and Mathematical Statistics, University of Cambridge, Cambridge, CB2 1SB, Fngland.

N. J. A. Sloane is with the Mathematical Sciences Research Center, Bell Laboratories, Murray Hill, NJ 07974.

IEEE Log Number 8406957
Two other results worth mentioning here are the following.

5) Several well-known codes unexpectedly turn out to be lexicographic codes, including Hamming codes and the binary Golay codes of length 23 and 24 (Section III-B).

6) The constant weight binary lexicode of length 24 , distance 8 and weight 8 is the Steiner system $S(5,8,24)$ (Theorem 12). By imposing an additional constraint on a constant weight lexicode (see Section IV-E), Ryba obtained an almost equally simple construction for the Steiner system $S(5,6,12)$ (Theorem 13). The corresponding game, called Mathematical Blackjack (or Mathieu's Vingt-et-un) is described at the end of Section IV-E.

7) A number of constant weight codes with minimal distance 10 and containing a record number of codewords are given in Table XIII.

Some of the game-theoretic aspects of this work are described in [1] and [2]. The relations between the theories of games and of lexicographic codes, and in particular the multiplicative theorem, underly some of the results in [1]. Iowever, most of the results are published here for the first time. This work may be regarded as a coding-theoretic analog of the laminated lattices described in [5], [6].

The paper is arranged as follows. The connections with game theory are discussed in Section II, unrestricted lexicodes are treated in Section III, and Section IV deals with constant weight and constrained lexicodes. Tables IV-VIII and XII give the parameters of a number of lexicodes.

\section{The Connections with Game Theory}

\section{A. Grundy's Game}

We begin by describing Grundy's game [1, p. 96], [9, p. 8], which is a characteristic example of the class of games to be considered. In Grundy's game the typical position

$$
P_{a}+P_{b}+P_{c}+\cdots
$$

consists of a number of heaps containing

$$
a, b, c, \cdots
$$

objects respectively. There are two players, who move alternately. A legal move is to split any heap into two strictly smaller heaps of distinct sizes, that is, to replace any term $P_{h}$ in (1) by $P_{l}+P_{j}$, where $0<i<h, 0<j<h$, $i \neq j$ and $i+j=h$. The first player who is unable to move loses. 


\section{B. Heap Games in General}

Grundy's game is an example of a heap game. The general game of this type may be taken to have certain atomic positions $P_{i}$ and general position

$$
P_{a}+P_{b}+P_{c} \cdots \text {. }
$$

The rules are specified by giving an arbitrary family of turning sets, a typical turning set being written

$$
\{h, i, j, \cdots\}, \quad \text { with } h>i>j>\cdots .
$$

The legal move is to replace any term $P_{h}$ in (2) by $P_{i}+P_{j}+\cdots$, provided that $\{h, i, j, \cdots\}$ is a turning set. For Grundy's game the turning sets are $\{3,2,1\},\{4,3,1\}$, $\{5,4,1\},\{5,3,2\},\{6,5,1\}, \cdots$.

There is a well-known theory of heap games, due to Sprague and Grundy [1], [2], [9], [19]. There is a function $G(P)$ (the nim-value, Grundy number, or G-value) assigning integer values to positions $P$, with the following properties:

0) A player wins by consistently moving to positions of $G$-value zero.

1) $G(P)=\operatorname{mex}\{G(Q), G(R), \cdots\}$, taken over all positions $Q, R, \cdots$ obtained from $P$ by a single move, where "mex" (or minimal excluded value) means "the smallest number (from $0,1,2,3, \cdots$ ) not among."

2) The $G$-value of a general position $P_{a}+P_{b}+\cdots$ is given by

$$
G\left(P_{a}+P_{b}+\cdots\right)=G\left(P_{a}^{*}\right) \oplus G\left(P_{b}\right) \oplus \cdots,
$$

where $\oplus$ is nim-addition. (The nim-sum of numbers $i, j, k, \cdots$ is obtained by writing them in binary and adding without carries, or in other words by forming the exclusive-or of their binary representations $[2, \mathrm{p}$. 51]. See Table II.)

The $G$-values of the atomic positions in Grundy's game are given by the following table $(G(n)$ is the $G$-value of a single heap of size $n$ ).

$\begin{array}{ccccccccc}n & 1 & 2 & 3 & 4 & 5 & 6 & 7 & 8 \\ G(n) & 0 & 0 & 1 & 0 & 2 & 1 & 0 & 2\end{array}$

The reader will easily verify for example that a single heap of size 4 , or two heaps of size 3 and 6 , are winning positions. ${ }^{1}$

\section{The Winning Code}

Alternatively, the general position $\sum_{i=0} n_{i} P_{i}$ in such a game may be represented by the integral vector

$$
\left(\cdots n_{3} n_{2} n_{1}\right)
$$

(or by the vector

$$
\left(\cdots n_{3} n_{2} n_{1} n_{0}\right)
$$

\footnotetext{
${ }^{1} G(n)$ has been calculated for $n \leq 10^{7}$, but it is not even known if it is eventually a periodic function of $n[1, \mathrm{pp} .96,111],[10],[11]$.
}

if the zero heaps play a significant role). However, since $x \oplus x=0$ for all $x$, the outcome of such a position depends only on the parities of the $n_{i}$. The winning strategy is therefore encapsulated in a certain binary code, consisting of all vectors

$$
\left(\cdots \zeta_{3} \zeta_{2} \zeta_{1}\right), \quad \text { where } \zeta_{i}=0 \text { or } 1,
$$

for which the nim-sum

$$
\sum \zeta_{i} G\left(P_{i}\right)=0 .
$$

We call this the winning code for the game.

For example, in Grundy's game the first few codewords and the corresponding winning positions are shown in Table I. A vector $\cdots \zeta_{3} \zeta_{2} \zeta_{1}$, where $\zeta_{i}=0$ or 1 , is in the

TABLE I

First Few CODEwords and CoRresponding Winning Positions in tiII Code for Grundy's Game

\begin{tabular}{cc}
\hline codeword & heap sizes \\
\hline$\ldots 0000000$ & 0 \\
$\ldots .0000001$ & 1 \\
$\ldots .0000010$ & 2 \\
$\ldots .0000011$ & 2,1 \\
$\ldots .0001000$ & 4 \\
$\ldots .0001001$ & 4,1 \\
$\ldots .0001010$ & 4,2 \\
$\ldots .0001011$ & $4,2,1$ \\
$\ldots .0100100$ & 6,3 \\
$\ldots 1000000$ & 7 \\
$\ldots$ & $\ldots$ \\
\hline
\end{tabular}

code if and only if

$$
\zeta_{1} G(1) \oplus \zeta_{2} G(2) \oplus \zeta_{3} G(3) \oplus \cdots=0,
$$

i.e.,

$$
\zeta_{3} \oplus 2 \zeta_{5} \oplus \zeta_{6} \oplus 2 \zeta_{8} \oplus \cdots=0 .
$$

Since the code is defined by the linear condition (3), we deduce the following surprising result.

Theorem 1: The winning code for a heap game is a linear code over GF (2).

\begin{tabular}{rrrrrrrr}
\hline 9 & 10 & 11 & 12 & 13 & 14 & 15 & $\ldots$ \\
1 & 0 & 2 & 1 & 3 & 2 & 1 & $\ldots$.
\end{tabular}

The codewords as just defined have infinitely many coordinates. However, for any $n$, we may obtain a code of length $n$ by restricting attention to words that vanish outside the last $n$ coordinates.

\section{Generalization to Base B; Lexicodes}

We now define analogs of these games (and codes) in which the number 2 is replaced by a general base $B$. Theorem 1 generalizes satisfactorily if $B$ is a power of 2 , but the codes seem to have little structure for other values of $B$.

In view of (3), we can regard the heap game described in Section II-B as played with binary numbers $N=\Sigma \zeta_{i} 2^{i}$, 
where $\zeta_{i}=0$ or 1 (or with the corresponding binary vectors $\left.\left(\cdots \zeta_{3} \zeta_{2} \zeta_{1}\right)\right)$, and the legal move is to replace $N$ by $N^{i}=\sum \zeta_{i}^{\prime} 2^{i}$ provided

1) $N^{\prime}<N$ (this is the lexicographic condition), and

2) the collection of $i$ such that $\zeta_{i}^{\prime} \neq \zeta_{i}$ is a turning set.

More generally, we may consider a game defined by giving a base $B$ and a family of finite turning sets of the form

$$
\{h, i, j, \cdots\}, \quad h>i>j>\cdots .
$$

A position is described by a number

$$
N=\sum \zeta_{i} B^{i}, \quad \zeta_{i}=0,1, \cdots, B-1,
$$

written in the base $B$, or equivalently by a vector

$$
N=\left(\cdots \zeta_{3} \zeta_{2} \zeta_{1}\right), \quad \zeta_{i}=0,1, \cdots, B-1 .
$$

Again the legal move is to replace $N$ by $N^{\prime}=\sum \zeta_{i}^{\prime} B^{i}$ provided conditions 1) and 2) are satisfied. Thus the turning sets only specify where two successive positions must differ, not by how much. The Sprague-Grundy theory also applies to these games, and we may define the corresponding winning code as in Section II-C.

For each family of turning sets and each base $B$ we may also define another code called an (unrestricted) lexicographic code, or lexicode. This code is defined by the following greedy algorithm. The possible words ( $\cdots$ $\left.\zeta_{3} \zeta_{2} \zeta_{1}\right), 0 \leq \zeta_{i}<B$, are considered in the lexicographic order determined by the corresponding number

$$
N=\sum \zeta_{i} B^{i} .
$$

A word is rejected if there is some earlier word $N^{\prime}=(\cdots$ $\left.\zeta_{3}^{\prime} \zeta_{2}^{\prime} \zeta_{1}^{\prime}\right)$, for which the set of $i$ with $\zeta_{i}^{\prime} \neq \zeta_{i}$ is a turning set, and is otherwise accepted, i.e., placed in the code. The set of coordinates $i$ where $N$ and $N^{\prime}$ differ will be denoted by $\Delta\left(N, N^{\prime}\right)$. It turns out that this code is the same as the winning code.

Theorem 2: For any turning set and any base, the winning moves in the game are to move to positions corresponding to the codewords in the lexicode.

Proof: The proof is by induction on $N$ (the position). There are two things to be checked. If $N$ is not in the lexicode, this must be because there is a smaller number $N^{\prime}$ in the lexicode for which $\Delta\left(N, N^{\prime}\right)$ is a turning set. Therefore, by the induction hypothesis, the move from $N$ to $N^{\prime}$ is a winning move, and $N$ is not a winning position. On the other hand, if $N$ is in the lexicode, and $N$ to $N^{\prime}$ is any legal move, then $N^{\prime}<N$ and $\Delta\left(N, N^{\prime}\right)$ is a turning set. Since we accept $N$ we must have rejected each such $N^{\prime}$, and so the move from $N$ to $N^{\prime}$ cannot be a winning move. Therefore $N$ is a winning position. This completes the proof.

\section{E. Examples}

Example 1: We take $B=8$ and let the turning sets be all sets of size 1 or 2 . Thus distinct codewords must differ in at least three places. Applying the greedy algorithm, we find that the lexicode contains the words

0000

0111

0222

0555

0777

1012

1103

Theorem 4 below shows that this code is closed under componentwise nim-addition of vectors. For example, $0555 \oplus 1103=1456$ will again bc in the code. (Table II contains a nim-addition table.)

Example 2: More generally, for any base $B$, if the turning sets consist of all sets of cardinality $1,2,3, \cdots, d-$ 1 , the corresponding lexicode is that with minimal Hamming distance $d$.

Example 3: For $B=d=4$, the lexicode begins

$$
\begin{aligned}
& 000000 \\
& 001111 \\
& 002222 \\
& 003333 \\
& 010123 \\
& 011032 \\
& 012301
\end{aligned}
$$

As in Example 1 this code is closed under nim-addition. But now Theorem 5 below shows that it is also closed under nim-multiplication by $0,1,2,3$, nim-multiplication being defined by

\begin{tabular}{|l|llll|}
\hline$\otimes$ & 0 & 1 & 2 & 3 \\
\hline 0 & 0 & 0 & 0 & 0 \\
1 & 0 & 1 & 2 & 3 \\
2 & 0 & 2 & 3 & 1 \\
3 & 0 & 3 & 1 & 2 \\
\hline
\end{tabular}

Thus $3 \otimes(010123)=(030312)$ will also be in the code. (Nim-multiplication of numbers greater than three is more subtle and is described in Section II-G.)

In fact if we stop at length 6 this code is the hexacode, the $[6,3,4]$ extended "Golay" code over GF $(4)=$ $\left\{0,1, \omega, \bar{\omega}=\omega^{2}=\omega+1\right\}$, where we identify $\omega$ with 2 and $\bar{\omega}$ with $3([4$, eq. (7)], [7]).

\section{F. The Additive Theorem}

By combining Theorems 1 and 2 we immediately obtain the following result.

Theorem 3: If $B=2$ the lexicode defined by any family of turning sets is a binary linear code.

This may be generalized.

Theorem 4-The Additive Theorem: If $B$ is a power of 2 the lexicode defined by any family of turning sets is closed under componentwise nim-addition. 
Proof: It will suffice to consider the case $B=8$, the general case being exactly similar. We convert octal vectors into binary vectors by replacing each octal digit $\zeta_{i}$ by three binary digits $\zeta_{3 i+2}, \zeta_{3 i+1}, \zeta_{3 i}$ in the usual way:

\begin{tabular}{cccc}
\hline$\zeta_{i}$ & $\zeta_{3 i+2}$ & $\zeta_{3 i+1}$ & $\zeta_{3 i}$ \\
\hline 0 & 0 & 0 & 0 \\
1 & 0 & 0 & 1 \\
2 & 0 & 1 & 0 \\
7 & 1 & $\cdots$ & \\
7
\end{tabular}

In this way the original octal game becomes a binary game in which $T$ is a turning set just if

$$
\left\{\left[\frac{i}{3}\right]: i \in T\right\}
$$

was a turning set in the octal game (where $[x]$ denotes the integer part of $x$ ). The desired result now follows by applying Theorem 3 to the new binary game.

Conversely, if $B$ is not a power of 2 then in general the lexicode is not closed under any reasonable definition of addition. For example, if $B=3$ and the turning sets are all the sets of cardinality 1 (i.e., the code has minimal distance 2) then the lexicode begins

0000
0011
0022
0101
0110
0202
$\ldots$..

However, the sum of the third and fourth words is not in the code.

\section{G. Nim-Multiplication}

In any additive group the rule for addition must have the property that if

$$
a \neq a^{\prime} \quad \text { and } \quad b \neq b^{\prime}
$$

then

$$
a+b \neq a^{\prime}+b \text { or } a+b^{\prime} .
$$

Nim-addition can be defined by setting the sum of $a$ and $b$ equal to the lexicographically earliest value permitted by this property. More precisely, the nim-sum $a \oplus b$ (defined in Section II-B) can also be defined recursively by

$$
a \oplus b=\operatorname{mex}_{a^{\prime}<, b^{\prime}<b}\left\{a^{\prime} \oplus b, a \oplus b^{\prime}\right\} .
$$

There is an operation $\otimes$ called nim-multiplication which together with $\oplus$ converts the integers into a field $[2, \mathrm{ch} .6]$. In any field if $a \neq a^{\prime}, b \neq b^{\prime}$, then

$$
\left(a-a^{\prime}\right)\left(b-b^{\prime}\right) \neq 0
$$

and so

$$
a b \neq a^{\prime} b+a b^{\prime}-a^{\prime} b^{\prime},
$$

or in a field of characteristic 2

$$
a b \neq a^{\prime} b+a b^{\prime}+a^{\prime} b^{\prime} .
$$

The nim-product of $a$ and $b$ is the lexicographically earliest value permitted by this property. More precisely, $a \otimes b$ is defined recursively by

$$
a \otimes b=\operatorname{mex}_{a^{\prime}<a, b^{\prime}<b}\left\{a^{\prime} \otimes b \oplus a \otimes b^{\prime} \oplus a^{\prime} \otimes b^{\prime}\right\}
$$

(where nim-multiplication takes precedence over nimaddition). It is a remarkable fact that $\oplus$ and $\otimes$ as defined by the greedy algorithms (5) and (6) convert the numbers $0,1,2,3, \cdots$ into a field $[2$, p. 55]. The characteristic of this field is 2 . Also, for all $a$, the numbers less than $2^{2^{a}}$ form a subfield isomorphic to the Galois field GF $\left(2^{2^{a}}\right)[2$, Theorem 49]. We have already seen an illustration of this in the case $B=4=2^{2^{1}}$ in Example 3 .

The nim-addition and nim-multiplication tables for numbers less than $16=2^{2^{2}}$ are given in Tables II and III [2, pp. 51,52]. In view of the previous remark, these numbers form the field GF (16).

TABLE II

Nim-Addition of Numbers 0 To 15

\begin{tabular}{r|rrrrrrrrrrrrrrrrrrrr}
\hline$\oplus$ & 0 & 1 & 2 & 3 & 4 & 5 & 6 & 7 & 8 & 9 & 10 & 11 & 12 & 13 & 14 & 15 \\
\hline 0 & 0 & 1 & 2 & 3 & 4 & 5 & 6 & 7 & 8 & 9 & 10 & 11 & 12 & 13 & 14 & 15 \\
1 & 1 & 0 & 3 & 2 & 5 & 4 & 7 & 6 & 9 & 8 & 11 & 10 & 13 & 12 & 15 & 14 \\
2 & 2 & 3 & 0 & 1 & 6 & 7 & 4 & 5 & 10 & 11 & 8 & 9 & 14 & 15 & 12 & 13 \\
3 & 3 & 2 & 1 & 0 & 7 & 6 & 5 & 4 & 11 & 10 & 9 & 8 & 15 & 14 & 13 & 12 \\
4 & 4 & 5 & 6 & 7 & 0 & 1 & 2 & 3 & 12 & 13 & 14 & 15 & 8 & 9 & 10 & 11 \\
5 & 5 & 4 & 7 & 6 & 1 & 0 & 3 & 2 & 13 & 12 & 15 & 14 & 9 & 8 & 11 & 10 \\
6 & 6 & 7 & 4 & 5 & 2 & 3 & 0 & 1 & 14 & 15 & 12 & 13 & 10 & 11 & 8 & 9 \\
7 & 7 & 6 & 5 & 4 & 3 & 2 & 1 & 0 & 15 & 14 & 13 & 12 & 11 & 10 & 9 & 8 \\
8 & 8 & 9 & 10 & 11 & 12 & 13 & 14 & 15 & 0 & 1 & 2 & 3 & 4 & 5 & 6 & 7 \\
9 & 9 & 8 & 11 & 10 & 13 & 12 & 15 & 14 & 1 & 0 & 3 & 2 & 5 & 4 & 7 & 6 \\
10 & 10 & 11 & 8 & 9 & 14 & 15 & 12 & 13 & 2 & 3 & 0 & 1 & 6 & 7 & 4 & 5 \\
11 & 11 & 10 & 9 & 8 & 15 & 14 & 13 & 12 & 3 & 2 & 1 & 0 & 7 & 6 & 5 & 4 \\
12 & 12 & 13 & 14 & 15 & 8 & 9 & 10 & 11 & 4 & 5 & 6 & 7 & 0 & 1 & 2 & 3 \\
13 & 13 & 12 & 15 & 14 & 9 & 8 & 11 & 10 & 5 & 4 & 7 & 6 & 1 & 0 & 3 & 2 \\
14 & 14 & 15 & 12 & 13 & 10 & 11 & 8 & 9 & 6 & 7 & 4 & 5 & 2 & 3 & 0 & 1 \\
15 & 15 & 14 & 13 & 12 & 11 & 10 & 9 & 8 & 7 & 6 & 5 & 4 & 3 & 2 & 1 & 0 \\
\hline
\end{tabular}

TABLE III

Nim-MUlTiplication OF NUMBERs 0 TO 15

\begin{tabular}{r|rrrrrrrrrrrrrrrrrrr}
\hline$\otimes$ & 0 & 1 & 2 & 3 & 4 & 5 & 6 & 7 & 8 & 9 & 10 & 11 & 12 & 13 & 14 & 15 \\
\hline 0 & 0 & 0 & 0 & 0 & 0 & 0 & 0 & 0 & 0 & 0 & 0 & 0 & 0 & 0 & 0 & 0 \\
1 & 0 & 1 & 2 & 3 & 4 & 5 & 6 & 7 & 8 & 9 & 10 & 11 & 12 & 13 & 14 & 15 \\
2 & 0 & 2 & 3 & 1 & 8 & 10 & 11 & 9 & 12 & 14 & 15 & 13 & 4 & 6 & 7 & 5 \\
3 & 0 & 3 & 1 & 2 & 12 & 15 & 13 & 14 & 4 & 7 & 5 & 6 & 8 & 11 & 9 & 10 \\
4 & 0 & 4 & 8 & 12 & 6 & 2 & 14 & 10 & 11 & 15 & 3 & 7 & 13 & 9 & 5 & 1 \\
5 & 0 & 5 & 10 & 15 & 2 & 7 & 8 & 13 & 3 & 6 & 9 & 12 & 1 & 4 & 11 & 14 \\
6 & 0 & 6 & 11 & 13 & 14 & 8 & 5 & 3 & 7 & 1 & 12 & 10 & 9 & 15 & 2 & 4 \\
7 & 0 & 7 & 9 & 14 & 10 & 13 & 3 & 4 & 15 & 8 & 6 & 1 & 5 & 2 & 12 & 11 \\
8 & 0 & 8 & 12 & 4 & 11 & 3 & 7 & 15 & 13 & 5 & 1 & 9 & 6 & 14 & 10 & 2 \\
9 & 0 & 9 & 14 & 7 & 15 & 6 & 1 & 8 & 5 & 12 & 11 & 2 & 10 & 3 & 4 & 13 \\
10 & 0 & 10 & 15 & 5 & 3 & 9 & 12 & 6 & 1 & 11 & 14 & 4 & 2 & 8 & 13 & 7 \\
11 & 0 & 11 & 13 & 6 & 7 & 12 & 10 & 1 & 9 & 2 & 4 & 15 & 14 & 5 & 3 & 8 \\
12 & 0 & 12 & 4 & 8 & 13 & 1 & 9 & 5 & 6 & 10 & 2 & 14 & 11 & 7 & 15 & 3 \\
13 & 0 & 13 & 6 & 11 & 9 & 4 & 15 & 2 & 14 & 3 & 8 & 5 & 7 & 10 & 1 & 12 \\
14 & 0 & 14 & 7 & 9 & 5 & 11 & 2 & 12 & 10 & 4 & 13 & 3 & 15 & 1 & 8 & 6 \\
15 & 0 & 15 & 5 & 10 & 1 & 14 & 4 & 11 & 2 & 13 & 7 & 8 & 3 & 12 & 6 & 9 \\
\hline
\end{tabular}

Nim-sums and products can be easily computed using the field laws and the facts that

- for $N$ of the form $2^{a}$ we have

$$
\begin{aligned}
& N \oplus n=N+n \quad \text { for } n<N, \\
& N \oplus N=0 ;
\end{aligned}
$$


- for $N$ of the form $2^{2^{a}}$ we have

$$
\begin{aligned}
& N \otimes n=N n \quad \text { for } n<N, \\
& N \otimes N=\frac{3}{2} N .
\end{aligned}
$$

The standard reference for nim-multiplication is [2, ch. 6]. See also [1, ch. 14], [14], and [15].

\section{H. The Multiplicative Theorem}

Theorem 5-The Multiplicative Theorem: If $B$ is of the form $2^{2^{4}}$ then the lexicode defined by any family of turning sets is closed under componentwise nim-multiplication by numbers $\alpha$ in the range $0 \leq \alpha<B$. In other words the lexicode is a linear code over the field GF $\left(2^{2^{u}}\right)$.

Before giving the proof, let us define $f(\zeta, P)$ to be the $G$-value of the position with a single $\zeta(0 \leq \zeta \leq B-1)$ in coordinate $P$ :

$$
f(\zeta, P)=G(\cdots 0,0, \zeta, 0, \cdots, 0),
$$

and let $f(P)=f(1, P)$.

Proof: By Theorem 2 the lexicode consists of the positions with $G$-value zero. Therefore by the additive theorem (Theorem 4), the desired conclusion will follow if we show that

$$
f(\zeta, P)=\zeta \otimes f(P)
$$

for all $\zeta, P$. We show this by a double induction on $\alpha$ and $P$. From Rule 1 of Section II-B,

$$
f(\zeta, P)=\operatorname{mex}_{(13)}\left\{f\left(\zeta^{\prime}, P\right) \oplus \sum f\left(\eta_{i}, Q_{i}\right)\right\}
$$

where the mex is taken over these values:

$$
\begin{aligned}
& 0 \leq \zeta^{\prime}<\zeta, \\
& \left\{P, Q_{1}, Q_{2}, \cdots\right\} \text { is a turning set, } \\
& 0<\eta_{i}<B \quad \text { for all } i .
\end{aligned}
$$

(Every coordinate of the turning set must be changed.) By the induction hypothesis,

$$
f(\zeta, P)=\operatorname{mex}\left\{\zeta^{\prime} \otimes f(P) \oplus \sum \eta_{i} \otimes f\left(Q_{i}\right)\right\} .
$$

On the other hand, from (6),

$$
\zeta \otimes f(P)=\operatorname{mex}\left\{\zeta^{\prime} \otimes f(P) \oplus\left(\zeta \oplus \zeta^{\prime}\right) \otimes \lambda\right\}
$$

where $\zeta^{\prime}<\zeta$ and $\lambda<f(P)$. Now

$$
f(P)=\operatorname{mex}_{(13)}\left\{\sum \eta_{i} \otimes f\left(Q_{i}\right)\right\}
$$

so all $\lambda<f(P)$ can be written in the form $\lambda=\sum \eta_{i} \otimes$ $f\left(Q_{i}\right)$. Therefore (15) becomes

$$
\begin{aligned}
\zeta & \otimes f(P) \\
& =\operatorname{mex}_{(13)}\left\{\zeta^{\prime} \otimes f(P) \oplus \sum\left(\zeta \oplus \zeta^{\prime}\right) \otimes \eta_{i} \otimes f\left(Q_{i}\right)\right\} .
\end{aligned}
$$

The numbers less than $B$ form a field, $\operatorname{GF}(B)$, and therefore (since $\alpha \oplus \alpha^{\prime}$ is a nonzero constant), the sum in (16) is equal to $\sum \eta_{i} \otimes f\left(Q_{i}\right)$. Equations (14) and (16) now agree, which establishes the desired result.

Conversely, if $B$ is not of the form $2^{2^{a}}$, then in general the lexicode is not closed under any reasonable definition of multiplication. This may be seen for example in the case $B=8$ and $d=3$; we omit the details.

\section{LEXICODES}

\section{A. Introduction}

In this section we discuss some particular families of lexicodes in morc detail. We specify the base $B$, the desired minimal Hamming distance $d$, and take the turning sets to consist of all sets of cardinality $1,2, \cdots, d-1$. Then the lexicode is formed by starting with the zero word and repeatedly adjoining the lexicographically earliest word that is at Hamming distance at least $d$ from all previous words.

As we have seen, if $B=2^{a}$ the lexicode is closed under addition (Theorem 4), and if $B=2^{2^{4}}$ it is also closed under multiplication by scalars, i.e., is a linear code over GF ( $B)$ (Theorem 5).

A code of length $n$ is obtained by accepting only those codewords that vanish outside the last $n$ coordinates.

The parameters of the lexicodes are summarized in Tables IV-VII. Tables IV, V, and VI give the number of codewords in the lexicodes with $d=3,4$, and 6 , respectively, for various bases and lengths. Table VII gives the dimension $k$ of the binary $(B=2)$ lexicodes for $n \leq 44$ and $d \leq 10$. (In view of Example 3 ) in Section III-B, it is enough to consider even values of $d$.)

\section{TABLE IV}

Number of Codewords ${ }^{\mathrm{a}}$ IN LEXICODE OF BASE $B$, LeNGTH $n$ and Minimal Distance $d=3$

\begin{tabular}{r|rrrrrrrrrrrr}
\hline$n \backslash B$ & 2 & 3 & 4 & 5 & 6 & 7 & 8 & 9 & 10 & 15 & 16 & 17 \\
\hline 3 & 2 & 3 & 4 & 5 & 6 & 7 & 8 & 9 & 10 & 15 & 16 & 17 \\
4 & 2 & 9 & 16 & 17 & 22 & 25 & 32 & 48 & 70 & 187 & 256 & 257 \\
5 & 4 & 9 & 64 & 74 & 112 & 182 & $2^{8}$ & 372 & 532 & & & \\
6 & 8 & 24 & 64 & 265 & 618 & 1175 & $2^{11}$ & & & & & \\
7 & 16 & 72 & $2^{8}$ & 1113 & 2994 & & $2^{14}$ & & & & & \\
8 & 16 & 198 & $2^{10}$ & & & & $2^{17}$ & & & & & \\
9 & 32 & 519 & $2^{12}$ & & & & $2^{20}$ & & & & & \\
10 & 64 & 1390 & $2^{14}$ & & & & $2^{23}$ & & & & & \\
11 & 128 & 3650 & $2^{16}$ & & & & & & & & & \\
\hline
\end{tabular}

a The continuation of the $B=8$ column can be found in Section III-C.

TABLE V

Number of CODEWORDS IN LEXICODE OF BASE $B$, LeNGTH $n$ and Minimal Distance $d=4$

\begin{tabular}{r|rrrrrrrrr}
\hline$n \backslash B$ & 2 & 3 & 4 & 5 & 6 & 7 & 8 & 9 & 10 \\
\hline 4 & 2 & 3 & 4 & 5 & 6 & 7 & 8 & 9 & 10 \\
5 & 2 & 3 & 16 & 17 & 18 & 27 & 32 & 33 & 46 \\
6 & 4 & 10 & 64 & 67 & 88 & 147 & $2^{8}$ & 314 & 446 \\
7 & 8 & 24 & 64 & 165 & 390 & 766 & $2^{11}$ & & \\
8 & 16 & 60 & $2^{8}$ & 676 & & & $2^{14}$ & & \\
9 & 16 & 136 & $2^{10}$ & & & & $2^{16}$ & & \\
10 & 32 & 334 & $2^{12}$ & & & & $2^{19}$ & & \\
11 & 64 & 807 & $2^{14}$ & & & & $2^{22}$ & & \\
\hline
\end{tabular}


TABLE VI

Number of CODEWORdS IN LEXICODE OF BASE $B$, LENGTH $n$, and Minimal Distance $d=6$

\begin{tabular}{r|rrrrrrr}
\hline$n \backslash B$ & 2 & 3 & 4 & 5 & 6 & 7 & 8 \\
\hline 6 & 2 & 3 & 4 & 5 & 6 & 7 & 8 \\
7 & 2 & 3 & 4 & 5 & 12 & 25 & 32 \\
8 & 2 & 9 & 16 & 33 & 58 & 95 & 256 \\
9 & 4 & 17 & 64 & 99 & 222 & & \\
10 & 4 & 29 & 256 & & & & \\
11 & 8 & 59 & & & & & \\
12 & 16 & 124 & & & & & \\
13 & 16 & 269 & & & & & \\
\hline
\end{tabular}

TABLE VII

Binary Lexicodes. The Table gives the Dimension $k$ of BINARY $[n, k, d]$ LEXICODES FOR $n \leq 44$ AND $d \leq 10$

\begin{tabular}{r|rrrr|r|rrrr}
\hline$n \backslash d$ & 4 & 6 & 8 & 10 & $n \backslash d$ & 4 & 6 & 8 & 10 \\
\hline 4 & 1 & 0 & 0 & 0 & 25 & 19 & 14 & 12 & 7 \\
5 & 1 & 0 & 0 & 0 & 26 & 20 & 15 & 12 & 8 \\
6 & 2 & 1 & 0 & 0 & 27 & 21 & 16 & 12 & 9 \\
7 & 3 & 1 & 0 & 0 & 28 & 22 & 17 & 13 & 9 \\
8 & 4 & 1 & 1 & 0 & 29 & 23 & 18 & 13 & 10 \\
9 & 4 & 2 & 1 & 0 & 30 & 24 & 19 & 14 & 11 \\
10 & 5 & 2 & 1 & 1 & 31 & 25 & 19 & 15 & 12 \\
11 & 6 & 3 & 1 & 1 & 32 & 26 & 20 & 16 & 12 \\
12 & 7 & 4 & 2 & 1 & 33 & 26 & 21 & 16 & 13 \\
13 & 8 & 4 & 2 & 1 & 34 & 27 & 22 & 17 & 14 \\
14 & 9 & 5 & 3 & 1 & 35 & 28 & 23 & 18 & 14 \\
15 & 10 & 6 & 4 & 2 & 36 & 29 & 24 & 19 & 15 \\
16 & 11 & 7 & 5 & 2 & 37 & 30 & 25 & 20 & 16 \\
17 & 11 & 8 & 5 & 2 & 38 & 31 & 26 & 21 & 17 \\
18 & 12 & 9 & 6 & 3 & 39 & 32 & 27 & 22 & 17 \\
19 & 13 & 9 & 7 & 3 & 40 & 33 & 27 & 23 & 18 \\
20 & 14 & 10 & 8 & 4 & 41 & 34 & 28 & 23 & 19 \\
21 & 15 & 11 & 9 & 5 & 42 & 35 & 29 & 24 & 20 \\
22 & 16 & 12 & 10 & 5 & 43 & 36 & 30 & 25 & 21 \\
23 & 17 & 12 & 11 & 6 & 44 & 37 & 31 & 26 & 21 \\
24 & 18 & 13 & 12 & 6 & & & & & \\
& & & & & & & & &
\end{tabular}

TABLE VIII

Number of Codewords in Hamming Code of Base $B$, Length $n$ (USING THE FIRST COLUMN) AND IN EXTENDED HAMMING Code of Base $B$, Length $m$ (Using the Last Column)

\begin{tabular}{c|rrrrrrr|r}
\hline$n \backslash B$ & 2 & 3 & 4 & 5 & 7 & 8 & 9 & $m$ \\
\hline 3 & 2 & 3 & 4 & 5 & 7 & 8 & 9 & 4 \\
4 & 2 & 9 & 16 & 25 & 49 & 64 & 81 & 5 \\
5 & 4 & 9 & 64 & 125 & 343 & 512 & 729 & 6 \\
6 & 8 & 27 & 64 & 625 & $7^{4}$ & $8^{4}$ & $9^{4}$ & 7 \\
7 & 16 & 81 & $4^{4}$ & 625 & $7^{5}$ & $8^{5}$ & $9^{5}$ & 8 \\
8 & 16 & 243 & $4^{5}$ & $5^{5}$ & $7^{6}$ & $8^{6}$ & $9^{6}$ & 9 \\
9 & 32 & 729 & $4^{6}$ & $5^{6}$ & $7^{6}$ & $8^{7}$ & $9^{7}$ & 10 \\
10 & 64 & 2187 & $4^{7}$ & $5^{7}$ & $7^{7}$ & $8^{7}$ & $9^{8}$ & 11 \\
11 & 128 & 6561 & $4^{8}$ & $5^{8}$ & $7^{8}$ & $8^{8}$ & $9^{8}$ & 12 \\
\hline
\end{tabular}

Table VIII shows the number of codewords in extended and/or shortened Hamming codes for the range of lengths and bases covered by Tables IV and V. Comparison of these tables shows that lexicodes are as good as Hamming codes for $B=2^{2^{a}}$ (as we shall see in Theorem 6, lexicodes actually are Hamming codes in this case); they are usually slightly inferior to Hamming codes when $B=2^{a}$ (they are sometimes better, for example when $B=8, n=11, d=4$ ); and they are worse for other values of $B$.
If we just consider binary lexicodes, as in Table VII, comparison with the tables in [16, Appendix A] shows that the lexicodes are very good. In the range of Table VII they have dimensions within one or two of the best codes known and often have the same dimension (see the next section). We now discuss some of these codes in more detail.

\section{B. Some Well-Known Codes}

We first mention that some well-known codes are lexicodes. The proofs of the following assertions will either be given later, can be found in [1], or are straightforward verifications.

1) Zero-Sum Codes: For $d=2$ and any $B$, the lexicode of length $n$ is the zero-sum code, consisting of all vectors

$$
\left(\zeta_{n-1} \quad \zeta_{n-2} \cdots \zeta_{2} \quad \zeta_{1}\right)
$$

for which the nim-sum $\Sigma \zeta_{i}=0$. For example, this is the even-weight code in the binary case. We omit the easy proof by induction.

2) Hamming Codes: When $B=2$ and $d=3$, the turning sets have size 1 and 2, and the game is $\operatorname{nim}^{2}$ itself, the oldest and best known heap game [1, p. 430]. The corresponding lexicodes of length $n=2^{m}-1$ coincide with binary Hamming codes (see Theorem 6), and those of other lengths are shortened Hamming codes. Similarly when $B=2, d=4$, and $n=2^{m}$ we obtain extended Hamming codes. The heap game for $d=4$ is called Mock Turtles [1, p. 431].

3) Extended Binary Codes: When $B=2$ the lexicodes with $d$ even are obtained from the lexicodes with $d$ odd by adding an overall parity check. This is equivalent to the Mock Turtle Theorem [1, p. 432]. So for $B=2$ it is only necessary to consider even values of $d$. (This property does not hold for $B>2$.)

4) The Extended Quadratic Residue Code of Length 18: The lexicode with $B=2, d=6$, and $n=18$ is the $[18,9,6]$ binary extended quadratic residue code $[16$, p. 483]. The corresponding game is called Moebius [1, p. 434].

5) The Extended Golay Code: The lexicode with $B=2$, $d=8$, and $n=24$ is the $[24,12,8]$ binary Golay code. The corresponding game is called Mogul [1, p. 435].

6) Binary Codes with Distance 10: If $B=2$ and $d=10$, when $n=27$ or 31 the lexicodes have parameters $[27,9,10]$ and $[31,12,10]$, respectively. Codes with the same parameters were constructed by Hashim and Constantinides [12] and Piret [17]. The corresponding game is Moidores.

7) The Tetracode: Taking $B=d=3, n=4$ we obtain the $[4,2,3]$ code over GF (3) sometimes called the tetracode $[4$, p. 321], [7]. The nine codewords are

$\begin{array}{lll}0000 & 1012 & 2021 \\ 0111 & 1120 & 2102 \\ 0222 & 1201 & 2210 .\end{array}$

This code is "accidentally" linear over GF (3). (See Table XI for the continuation.)

\footnotetext{
${ }^{2}$ This version of nim is also called Turning Turtles [1, p. 429].
} 
8) The Hexacode: As already mentioned in Section II, Example 3 , when $B=d=4$ and $n=6$ we obtain the $[6,3,4]$ hexacode over GF (4).

\section{The Case $B=2^{a}$}

When $B=2^{a}$ the lexicode may be efficiently specified by giving the values of the function $f(\zeta, i)$ (the $G$-value of a position in which there is a single nonzero digit $\zeta$, $0 \leq \zeta \leq B-1$, in coordinate $i$ - see (11)). It is convenient to write $f(\zeta, i)$ in the base $B$.

For example when $B=8$ and $d=3$ the values of $f(\zeta, i)$ are shown in Table IX, written in octal. To illustrate how this table was obtained, we derive the entry $f(2,3)=$

TABLE IX

$G$-Values $f(\zeta, i)$ of Coordinate Positions for the C.ASE $B=8, d=3$. The $G$-Values Are Written in Octal.

\begin{tabular}{r|rrrrrrrr}
\hline$i \backslash \zeta$ & 0 & 1 & 2 & 3 & 4 & 5 & 6 & 7 \\
\hline 0 & 000 & 001 & 002 & 003 & 004 & 005 & 006 & 007 \\
1 & 000 & 010 & 020 & 030 & 040 & 050 & 060 & 070 \\
2 & 000 & 011 & 022 & 033 & 044 & 055 & 066 & 077 \\
3 & 000 & 012 & 023 & 031 & 100 & 112 & 123 & 131 \\
4 & 000 & 013 & 021 & 032 & 104 & 117 & 125 & 136 \\
5 & 000 & 014 & 042 & 056 & 101 & 115 & 143 & 157 \\
6 & 000 & 015 & 041 & 054 & 105 & 110 & 144 & 151 \\
7 & 000 & 016 & 045 & 053 & 107 & 111 & 142 & 154 \\
8 & 000 & 017 & 046 & 051 & 103 & 114 & 145 & 152 \\
9 & 000 & 024 & 043 & 067 & 102 & 126 & 141 & 165 \\
10 & 000 & 025 & 047 & 062 & 200 & 225 & 247 & 262 \\
\hline
\end{tabular}

023. From the position

$$
\left(\begin{array}{cccccc} 
& 4 & 3 & 2 & 1 & 0 \\
& 0 & 2 & 0 & 0 & 0
\end{array}\right)
$$

we can move to any of

$$
\begin{aligned}
& \left(\begin{array}{llllll}
\cdots & 0 & x & 0 & 0 & y
\end{array}\right) \\
& \left(\begin{array}{llllll}
\cdots & 0 & x & 0 & y & 0
\end{array}\right) \\
& \left(\begin{array}{llllll}
\cdots & 0 & x & y & 0 & 0
\end{array}\right)
\end{aligned}
$$

where $x=0,1, y=0,1, \cdots, 7$ (since the turning sets are of size 1 and 2 ). So $f(2,3)$ is the mex of

$$
\left\{\begin{array}{l}
f(0,3) \oplus a b c=000 \oplus a b c \\
f(1,3) \oplus a b c=012 \oplus a b c
\end{array}\right.
$$

where $a b c=f(y, 0), f(y, 1)$ or $f(y, 2)$ is any entry from the first three rows of Table IX. It is easily checked that the first octal number not of the form (17) is 023 .

The additive property (Theorem 4) implies that the columns for $\zeta-1,2$, and 4 determine the others. The typical entry in the $\zeta=6$ column for example is the nim-sum of the entries in columns 2 and 4.

By Theorem 2, the codewords are the positions of $G$-value zero. We illustrate how the codewords are found from the $f(\zeta, i)$ table by an example. For what values of $x$ and $y$ is

a codeword? Since

$$
\cdots 0 \cdots 020 x y
$$

$$
f(2,3)=023 \quad f(x, 1)=0 x 0 \quad f(y, 0)=00 y
$$

(from Table IX), the answer is $x=2, y=3$, i.e.,

$$
\text { ․ } 002023 \text {. }
$$

In this way, if $d$ is small, it is easy to obtain the number of codewords and a basis directly from the table. The codes in the $B=8$ column of Table IV and V were found in this way.

These codes are comparable in efficiency with Reed-Solomon codes and, of course, exist for all lengths (whereas Reed-Solomon codes only exist for lengths up to $B+1$ or $B+2[16$, p. 317]). For example, when $d=3$, $B=8$, if the lexicode contains $2^{k_{l}}$ codewords and the Reed-Solomon code contains $2^{k_{R}}$ codewords, we have

$$
\begin{array}{rrrrrrrrrrrrrrr}
n & 3 & 4 & 5 & 6 & 7 & 8 & 9 & 10 & 11 & 12 & 13 & 14 & 15 & 16 \\
k_{l} & 3 & 5 & 8 & 11 & 14 & 17 & 20 & 23 & 25 & 28 & 31 & 34 & 37 & 40 \\
k_{R} & 3 & 6 & 9 & 12 & 15 & 18 & 21 . & & & & & & &
\end{array}
$$

(This is a continuation of the $B=8$ column in Table IV.)

\section{The Case $B=2^{2^{a}}$}

The multiplicative property of Theorem 5 makes it even easier to construct the lexicode in the case when $B$ is of the form $2^{2^{a}}$, since (12) holds. This property was used in calculating the extensive table of $G$-values for the case $B=2$ given in [1, p. 433]. Table VII was then derived from that table. One can also use (12) to establish the following result.

Theorem 6: In the case $B=2^{2^{a}}, d=3$, lexicodes of length

$$
n=1+B+B^{2}+\cdots+B^{m-1}=\frac{B^{m}-1}{B-1}
$$

are Hamming codes, and those of other lengths are shortened Hamming codes.

We omit the proof.

As mentioned in Example 3) above, the lexicode with $B=2$ and $d$ even is obtained by adding an overall parity check to the lexicode with $B=2$ and distance $d-1$. So as far as the earliest families of lexicodes are concerned, we have a complete theory in the cases $B$ arbitrary, $d=2$ (zero-sum codes-Example 1), $B=2, \quad d=3$ (binary Hamming codes-Example 2), $B=2, d=4$ (extended binary Hamming codes-Example 2), and $B=4, d=3$ (Hamming codes over GF (4),-Theorem 6).

The structure of the next family of lexicodes, for $B=d$ $=4$, has been determined by Wilson [22]. Let us define a Wilson number to be one whose base 4 expansion is either 1 or 10 , or else has the form $\zeta_{m} \quad \zeta_{m-1} \cdots \zeta_{2} \zeta_{1} \zeta_{0}$ (for $m \geq 2$ ) where

$$
\begin{aligned}
\zeta_{m} & =1, \\
\zeta_{i} & =0 \text { or } 1 \quad \text { for } m>i \geq 2, \\
\zeta_{1} & =0,1,2 \text { or } 3, \\
\zeta_{0} & =1+\zeta_{1}^{2}+\zeta_{2}^{2}+\cdots+\zeta_{m}^{2},
\end{aligned}
$$

where the addition and multiplication in (18) are in the nim sense. The first few Wilson numbers (written in base 
4) are

$$
\begin{aligned}
& 1,10,100,111,123,132,1000,1011,1023, \\
& 1032,1101,1110,1122,1133,10000, \cdots .
\end{aligned}
$$

Theorem 7 (Wilson [22]): The $G$-value $(f(1, i))$ of the position $\cdots 0,1,0, \cdots, 0$ (with a single 1 in the $i$ th coordinate) is the $i$ th Wilson number.

Thus $G(\cdots 0001)=1, G(\cdots 0010)=10, G(\cdots 0100)$ $=100, G(\cdots 1000)=111$, etc. (in base 4). For completeness we include a proof in our terminology.

Important Note: With the single exception that $4^{i}$ means the usual $i$ th power of 4 , all additions and multiplications in the proof are in the nim sense.

Proof: The proof is by induction. Suppose we have checked that the first $k G$-values are the first $k$ Wilson numbers $W_{0}, \cdots, W_{k-1}$. We must show that the next number

$$
n=\sum_{i=0}^{m} 4^{i} \delta_{i}
$$

that is not a linear combination of two of $W_{0}, \cdots, W_{k-1}$ is the next Wilson number $W_{k}$.

1) If some $\delta_{i}(i \geq 2)$ in (19) is 2 or 3 then $n$ is a linear combination of two of $W_{0}, \cdots, W_{k-1}$. For we may obtain $n$ as

$$
N=2 p+3 q
$$

where

$$
\begin{aligned}
& p=\sum_{i=2}^{m} 4^{i} \alpha_{i}+4 \alpha+\left(\alpha^{2}+\theta_{1}\right), \\
& q=\sum_{i=2}^{m} 4^{i} \beta_{i}+4 \beta+\left(\beta^{2}+\theta_{2}\right), \\
& \theta_{i}=\sum_{i=2}^{m} \alpha_{i}^{2}+1, \quad \theta_{2}=\sum_{i=2}^{m} \beta_{i}^{2}+1,
\end{aligned}
$$

and where we choose $\alpha_{i}$ and $\beta_{i}$ according to

$$
\begin{array}{lllll}
d_{i} & 0 & 1 & 2 & 3 \\
\alpha_{i} & 0 & 1 & 1 & 0 \\
\beta_{i} & 0 & 1 & 0 & 1
\end{array}
$$

for $m \geq i \geq 2$. Then $\alpha$ and $\beta$ are uniquely determined by

$$
\begin{aligned}
2 \alpha+3 \beta & =\delta_{1}, \\
2 \alpha^{2}+3 \beta^{2} & =\delta_{0}+2 \theta_{1}+3 \theta_{2} .
\end{aligned}
$$

Since we are working in the field GF (4), (21) and (22) can be solved for $\alpha$ and $\beta$. It is easy to check that, with these values of $p$ and $q, n$ is given by (20). $p$ and $q$ are Wilson numbers, and, since some $\delta_{i}(i \geq 2)$ is 2 or $3, p \neq q$.

2) We next check that no Wilson number is a linear combination of two earlier Wilson numbers. Suppose on the contrary that $n=a p+b q$, where $n$ is given by (19) and

$$
p=\sum_{i=0}^{m} 4^{i} \alpha_{i} \quad q=\sum_{i=0}^{m} 4^{i} \beta_{i}
$$

There are two cases. a) If $\alpha_{m}=\beta_{m}=1$, we must have $\{a, b\}=\{2,3\}$, say $a=2, b=3$. Since $\delta_{m-1}, \cdots, \delta_{2}$ are 0 or 1 , we must have $\alpha_{m-1}=\beta_{m-1}, \cdots, \alpha_{2}=\beta_{2}$. Then

$$
\begin{aligned}
\delta_{0} & =\sum_{i=1}^{m-1} \delta_{i}^{2}=\sum_{i=1}^{m-1}\left(2 \alpha_{i}+3 \beta_{i}\right)^{2} \\
& =3 \sum_{i=1}^{m-1} \alpha_{i}^{2}+2 \sum_{i=1}^{m-1} \beta_{i}^{2} \\
& =3 \alpha_{0}+2 \beta_{0} .
\end{aligned}
$$

But also $\delta_{0}=2 \alpha_{0}+3 \beta_{0}$, implying $\delta_{0}=\alpha_{0}=\beta_{0}$. Therefore $\alpha_{1}=\beta_{1}$, hence $p=q$, so $n=p=q$, contradicting $n>p$. b) If $\alpha_{m}=1, \beta_{m}=0$, we must have $a=1, b=0$, and then

$$
\begin{aligned}
\delta_{0} & =\alpha_{m}^{2}+\cdots+\left(\alpha_{1}+\beta_{1}\right)^{2} \\
& =\left(\alpha_{m}^{2}+\cdots+\alpha_{1}^{2}\right)+\left(1^{2}+\cdots+\beta_{1}^{2}\right) \\
& =\left(\alpha_{0}+1\right)+\beta_{0},
\end{aligned}
$$

which contradicts $\delta_{0}=\alpha_{0}+\beta_{0}$.

3) If all $\delta_{i}(i \geq 2)$ in (19) are 0 or $1, n$ has the form

$$
n=\sum_{i=2}^{m} 4^{i} \delta_{i}+4 \zeta+\xi \quad\left(\delta_{i}=0 \text { or } 1\right)
$$

We must show that the next numbers of this form that are not linear combinations of two earlier Wilson numbers are Wilson numbers. In other words, we must show that if the number of 1's in $\left\{\delta_{m}, \delta_{m-1}, \cdots, \delta_{2}\right\}$ is odd (resp. even) then $(\zeta, \xi)$ runs through

$$
\left.\begin{array}{l|l}
0,0 \\
1,1 \\
2,3 \\
3,2
\end{array} \quad \text { resp. } \begin{array}{r}
0,1 \\
1,0 \\
2,2 \\
3,3
\end{array}\right) .
$$

Let

$$
n^{\prime}=\sum_{i=2}^{m} 4^{i} \delta_{i}
$$

If the number of 1 's in $n^{\prime}$ is odd, then the earliest numbers of the form (23) that are accepted are

$$
p=n^{\prime}+4 \otimes 0+0
$$

(this is a Wilson number and by part 2) it is accepted), and

$$
q=n^{\prime}+4 \otimes 1+1
$$

(this is the smallest number that differs from $p$ in two coordinates). Then

$$
n^{\prime}+4 \otimes 2+2=3 p+2 q
$$

is excluded, but

$$
\begin{aligned}
& r=n^{\prime}+4 \otimes 2+3, \\
& s=n^{\prime}+4 \otimes 3+2
\end{aligned}
$$

are accepted. On the other hand, suppose the number of 1's in $n^{\prime}$ is even. We can write $n^{\prime}=n^{\prime \prime} \oplus n^{\prime \prime \prime}$, where the 
numbers of 1's in $n^{\prime \prime}$ and $n^{\prime \prime}$ are odd. Then

$$
\left(n^{\prime \prime} \text { or } n^{\prime \prime \prime}\right)+\left\{\begin{array}{l}
4 \otimes 0+0 \\
4 \otimes 1+1 \\
4 \otimes 2+3 \\
4 \otimes 3+2
\end{array}\right.
$$

are earlier Wilson numbers. Adding, we see that

$$
n^{\prime}+\left\{\begin{array}{l}
4 \otimes 0+0 \\
4 \otimes 1+1 \\
4 \otimes 2+3 \\
4 \otimes 3+2
\end{array}\right.
$$

are excluded, and so we must go to at least

$$
\begin{aligned}
& p=n^{\prime}+4 \otimes 0+1 \\
& q=n^{\prime}+4 \otimes 1+0 \\
& r=n^{\prime}+4 \otimes 2+2 \\
& s=n^{\prime}+4 \otimes 3+3 .
\end{aligned}
$$

Since these are Wilson numbers, by part 2) they are accepted. This completes the proof.

Corollary 8: For $B=d=4$, the lexicode of length $n=$ $2^{m}-2$ contains $4^{k}$ codewords, where $k=2^{m}-m-2$.

The case $m=3$ is described in Example 8 above.

Wilson's theorem does not hold for $B=2^{2^{a}}>4$ and $d=4$. The first few $G$-values $f(1, i)$ are shown in Table X, and the last entry in the table does not have the form of a Wilson number.

TABLE X

$G$-values $f(1, i)$ FOR CASE $B=2^{2^{a}}>4, d=4$. Here $\alpha$ Is ANY Digir $0,1, \cdots, B-1$, AND ALl ADDITIONS AND Multiplications ARE IN THE Nim SENSE

$$
\begin{gathered}
1 \\
B \\
B^{2}+\alpha B+\alpha \\
B^{3}+\alpha B+\alpha^{2} \\
B^{3}+B^{2}+\alpha B+\alpha^{2}+1 \\
B^{4}+\alpha B+\alpha^{2} \\
B^{4}+B^{2}+\alpha B+\alpha^{2}+1 \\
B^{4}+B^{3}+B^{2}+\alpha B+\alpha^{2} \\
B^{4}+2 B^{3}+4 B^{2}
\end{gathered}
$$

\section{E. Other Values of $B$}

Lexicodes in general appear to have little or no structure. This can already be seen in the case $B=d=3$. The numbers of codewords in the first few codes are given in Table IV, and the complete code of length 8 is shown in Table XI. We have been unable to discover any structure to this code (or the solution of the corresponding game).

\section{Constant Weight Lexicographic Codes}

\section{A. Introduction}

A constant weight lexicode is defined similarly: we consider all words of the specified weight in lexicographic order and accept a word if it is at Hamming distance at
TABLE XI

THE 198 Words of THE LeXICODE OF LENGTH 8, Distance $d=3$, AND BASE $B=3$

\begin{tabular}{llllll}
\hline 00000000 & 01100010 & 02201020 & 10221212 & 12121022 & 21020101 \\
00000111 & 01100101 & 02202012 & 11000001 & 12172712 & 21021022 \\
00000222 & 01101002 & 02202121 & 11000110 & 12201001 & 21022211 \\
00001012 & 01101220 & 02210220 & 11001020 & 12201122 & 21100202 \\
00001120 & 01102122 & 02211112 & 11001102 & 12202210 & 21102100 \\
00001201 & 01102211 & 02212202 & 11001211 & 12210011 & 21120221 \\
00002021 & 01120112 & 10010010 & 11002012 & 12212022 & 21122012 \\
00002102 & 01121011 & 10010101 & 11002121 & 12220201 & 21200111 \\
00002210 & 01121100 & 10011002 & 11002200 & 20010021 & 21200220 \\
00110001 & 01122020 & 10011220 & 11110000 & 20010212 & 21210102 \\
00110110 & 01122202 & 10012122 & 11110111 & 20011111 & 21212121 \\
00111020 & 01200022 & 10012211 & 11110222 & 20012100 & 21221201 \\
00111102 & 01201110 & 10020112 & 11111012 & 20020002 & 22000011 \\
00111211 & 01202000 & 10020221 & 11111120 & 20020120 & 22001000 \\
00112012 & 01211021 & 10021011 & 11111201 & 20021210 & 22001112 \\
00112121 & 01211200 & 10021100 & 11112021 & 20101021 & 22001221 \\
00112200 & 01221122 & 10022020 & 11112102 & 20102201 & 22002101 \\
00120022 & 01222210 & 10022202 & 11112210 & 20110220 & 22011120 \\
00210122 & 02012000 & 10100002 & 11200212 & 20120011 & 22012012 \\
00220010 & 02012221 & 10100120 & 11220002 & 20121000 & 22020222 \\
00220101 & 02020012 & 10100211 & 11220120 & 20121112 & 22022021 \\
00221002 & 02021001 & 10101010 & 11221010 & 20122222 & 22022200 \\
00221220 & 02021110 & 10101101 & 11222101 & 20200012 & 22100110 \\
00222112 & 02022122 & 10101222 & 11222222 & 20200100 & 22102022 \\
01010002 & 02100021 & 10102112 & 12000022 & 20201211 & 22110002 \\
01010120 & 02100200 & 10120200 & 12010200 & 20202020 & 22110121 \\
01010211 & 02101111 & 10122001 & 12011021 & 20211001 & 22111010 \\
01011010 & 02110212 & 10200021 & 12011212 & 20211222 & 22112211 \\
01011101 & 02120120 & 10201200 & 12012110 & 20212210 & 22121202 \\
01011222 & 02121221 & 10210202 & 12102000 & 21000122 & 22220000 \\
01012112 & 02122101 & 10211110 & 12102221 & 21012001 & 22220112 \\
01020021 & 02200102 & 10212000 & 12120010 & 21012220 & 22221011 \\
01020200 & 02200211 & 10221121 & 12120102 & 21020010 & 22222120 \\
\hline
\end{tabular}

least $d$ from all previously accepted words. Only binary codes will be considered here. The Hamming distance $d$ is necessarily even. To distinguish these codes from the lexicodes of Section III we refer to the latter as unrestricted lexicodes.

The game corresponding to a constant weight lexicode of weight $w$ and minimal distance $d=2 t \geq 4$ is the following. The typical position is a set $\left\{a_{1}, a_{2}, \cdots, a_{w}\right\}$ of distinct nonnegative integers, and the legal move is to decrease $1,2, \cdots$, or $t-1$ of these integers while preserving their distinctness. As always, the first player who is unable to move loses. (These games are no longer well described by turning sets.) We define the corresponding winning code as in Section II-C.

Theorem 9: For any $d=2 t \geq 4$ and any $w$, the winning code for this game is the constant weight lexicode with the same parameters.

The proof is analogous to that of Theorem 2 and is omitted.

\section{B. The Case $d=2$}

The condition $d=2$ is automatically satisfied, and the corresponding lexicode consists of all codewords of weight $w$.

\section{The Case $d=4$}

The constant weight lexicode for $d=4$ and any $w$ is the winning code for Welter's game [1, pp. 472-481], [2, ch. 
13], [20], [21]. This is the only other case (besides $d=2$ ) where a complete theory exists.

Welter's game is the case $t=2$ of the game described in Section IV-A. A typical position is a set $\left\{a_{1}, a_{2}, \cdots, a_{w}\right\}$ of distinct nonnegative integers, and the legal move is to decrease one of these integers while preserving their distinctness. The complete solution [1, pp. 472-481], [2, ch. 13] uses Welter's remarkable function $W\left(a_{1}, a_{2}, \cdots, a_{w}\right)$, which can be defined recursively by

$$
\begin{aligned}
W\left(a_{1}\right) & =a_{1}, \\
W\left(a_{1}, a_{2}\right) & =\left(a_{1} \oplus a_{2}\right)-1
\end{aligned}
$$

and

$$
\begin{aligned}
& W\left(a_{1}, \cdots, a_{k+1}\right) \\
& =W\left(a_{2}, \cdots, a_{k}\right) \\
& \quad \oplus\left(\left(W\left(a_{1}, \cdots, a_{k}\right) \oplus W\left(a_{2}, \cdots, a_{k+1}\right)\right)-1\right) .
\end{aligned}
$$

The -1 in (25) is ordinary subtraction, so the definition of $W$ mixes nim-addition and ordinary subtraction.

Theorem 10: $\left(a_{1}, \cdots, a_{w}\right)$ is a winning position for Welter's game, or equivalently, the vector with 1 's in positions $a_{1}, \cdots, a_{w}$ is in the lexicode with constant weight $w$ and minimal distance 4 , if and only if

$$
W\left(a_{1}, \cdots, a_{w}\right)=0 .
$$

As we shall see, Theorem 10 is a consequence of the following property of Welter's function.

Theorem 11-The Even Alteration Property: If $W\left(a_{1}, \cdots, a_{w}\right)=n$, and $n^{\prime} \neq n$, there are unique nonnegative numbers $a_{1}^{\prime}, \cdots, a_{w}^{\prime}$ such that $a_{1}, \cdots, a_{w}, a_{1}^{\prime}, \cdots, a_{w}^{\prime}$ are distinct and satisfy

$$
\begin{gathered}
W\left(a_{1}^{\prime}, a_{2}, \cdots, a_{w}\right)=n^{\prime}, \\
W\left(a_{1}, a_{2}^{\prime}, \cdots, a_{w}\right)=n^{\prime}, \\
\cdots \\
W\left(a_{1}, a_{2}, \cdots, a_{w}^{\prime}\right)=n^{\prime} .
\end{gathered}
$$

More generally,

$$
W\left(a_{1}, a_{2}, \cdots, a_{w}\right)=n
$$

remains true if any even number of the letters $a_{1}, \cdots, a_{w}, n$ are replaced by the corresponding primed letters. Furthermore, an even number of the inequalities

are true.

$$
\begin{gathered}
a_{1}^{\prime}<a_{1}, \\
a_{2}^{\prime}<a_{2}, \\
\quad \cdots \\
a_{w}^{\prime}<a_{w}, \\
n^{\prime}<n
\end{gathered}
$$

For the proof see [2, ch. 13]. When $n \neq 0$, if we take $n^{\prime}=0$ we see that there is always at least one $a_{i}^{\prime}<a_{i}$, and so it is always possible to move from a position in which $W\left(a_{1}, \cdots, a_{w}\right) \neq 0$ to a position in which $W\left(a_{1}, \cdots, a_{w}\right)$ $=0$. Theorem 10 follows.

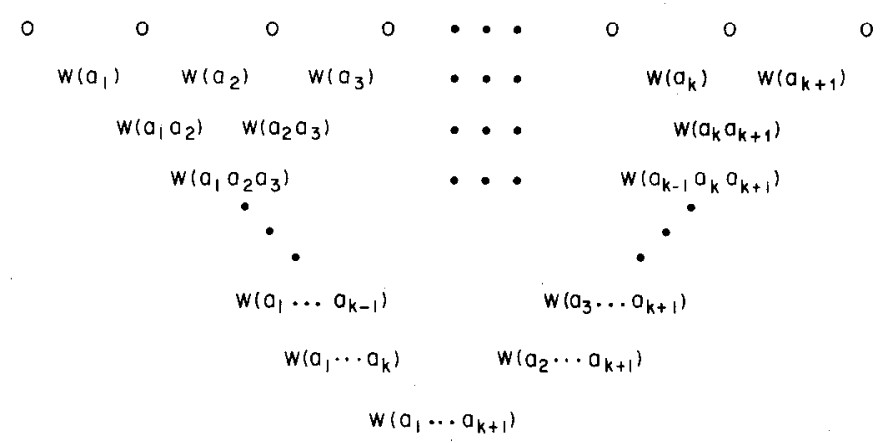

Fig. 1. Tableau for calculation of Welter's function. Entries are calculated by $W\left(a_{i}\right)=a_{i}$ and frieze rule (26).

The calculation of Welter's function from (25) is best carricd out using the tablcau shown in Fig. 1 (cf. [1, p. $476])$. Notice that once the first two rows are filled in (using (25a)), the tableau may be completed by the rule that any four entries arranged in a diamond

$$
{ }_{B} \stackrel{A}{ }{ }^{A} C
$$

must satisfy

$$
A \oplus D=(B \oplus C)-1 .
$$

Remark 1: A tableau satisfying such a rule is called a frieze pattern. Such patterns also have interesting properties if $x \oplus y$ is replaced by $x+y$ or $x y$; see [1, p. 475], [3],

Remark 2: Although it is not apparent from (25), Welter's function is a symmetric function of its arguments. For this and other combinatorial properties see [2, ch. 13].

Theorem 9 guarantees that if a vector $\zeta$ is not in the code, a codeword exists within Hamming distance 3 that is earlier than $\zeta$ in the lexicographic order. To find a winning move $a_{i} \rightarrow a_{i}^{\prime}$, and hence to decode $\zeta$, it is again convenient to use a frieze pattern (cf. [1, p. 477]).

We illustrate the decoding (or winning) strategy with an example. Suppose $w=5$, we are given $a, b, c, d, e$ and $n, n^{\prime}$, and wish to find $a^{\prime}, b^{\prime}, c^{\prime}, d^{\prime}, e^{\prime}$ as in Theorem 11. That theorem implies that if we place the numbers $a, b, \cdots, d^{\prime}, e^{\prime}$ in the first row of the tableau, and the numbers $n, n^{\prime}, n, n^{\prime}, \cdots$ in the fifth row, as in Fig. 2 , the frieze rule (26) will still hold. So we may compute $a^{\prime}, b^{\prime}, c^{\prime}, d^{\prime}, e^{\prime}$ by working downwards in the left half of the tableau and upwards in the right half, as illustrated by the numerical example in Fig. 3. Here $a=2, b=3, c=5$, $d=7, e=11, n=4$, and $n^{\prime}=0$. Fig. 3 shows that the winning move is from $\{2,3,5,7,11\}$ to $\{1,2,3,7,11\}$, i.e., that

$$
\cdots 100010101100
$$

should be decoded as

$$
\cdots 100010001110 \text {. }
$$

For $d=4, w=3$, and $n=2^{m}-1$, the words in the constant weight lexicode are the vectors of weight 3 in the Hamming code of length $2^{m}-1$. For $d=w=4$ and $n=2^{m}$, the lexicode consists of weight 4 vectors in the extended Hamming code of length $2^{m}$. These two asser- 


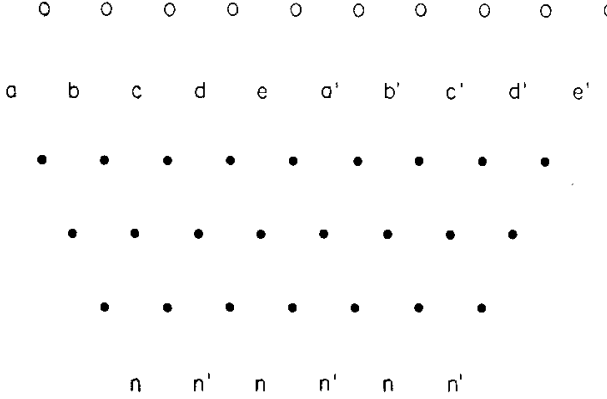

Fig. 2. Tableau for decoding.

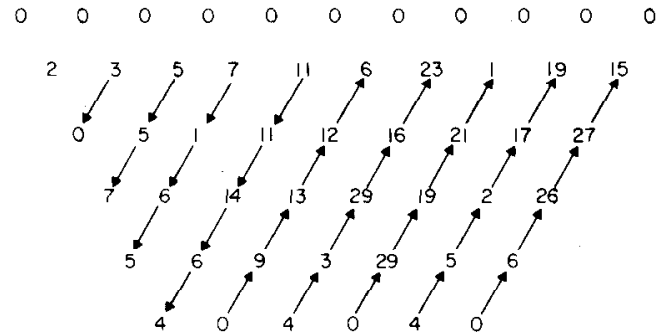

Fig. 3, Illustration of decoding technique in case $w=5$. Arrows indicate order in which entries are computed, using (26).

tions follow from the fact that Welter's game for $w=3$ and 4 is equivalent to nim [1, p. 473].

Table XII gives the number of codewords in the first few constant weight lexicodes with $d=4$. These values are of course lower bounds on the quantity $A(n, 4, w)$ where $A(n, d, w)$ is the size of the largest possible code of length $n$, constant weight $w$, and minimal distance $d$. However, in this case (for $d=4$ ), these codes are in general inferior to codes already known (compare [8, Table I]).

TABLE XII

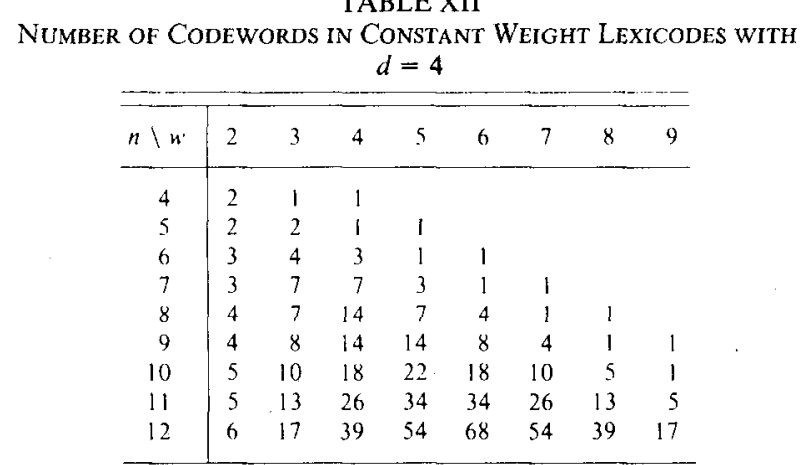

\section{Other Values of $d ; S(5,8,24)$}

In Section IV-C we saw that Welter's game gives the structure of constant weight lexicodes with minimal distance 4 . We have made extensive computations for other minimal distances, but although there are many interesting special cases, the resulting codes apparently display no general structure.

One interesting case occurs when $w=d=8$. We recall from Example 5) of Section III-B that the unrestricted lexicode for base $B=2, d=8$, and length 24 is the binary
Golay code containing

$\begin{array}{rlr}1 & \text { word of wcight } & 0 \\ 759 & \text { words of weight } & 8 \\ 2576 & \text { words of weight } & 12 \\ 759 & \text { words of weight } & 16 \\ 1 & \text { word of weight } & 24 .\end{array}$

Our computations show that at length 24 the constant weight lexicode with $w=d=8$ consists precisely of the 759 weight 8 codewords in the full code. Thus we have the following surprising fact.

Theorem 12: The words of the constant weight lexicode with $w=d=8$ and $n=24$ are the blocks (or octads) of a Steiner system ${ }^{3} S(5,8,24)$.

However, for $w=12$ and $d=8$, there are only 481 codewords of length 24, instead of the 2576 in the Golay code. For $w=16$ and $d=8$, the constant weight lexicode does indeed contain 759 codewords. However, they are not precisely the 759 weight 16 words of the unrestricted $d=8$ lexicode, but rather these words changed by the coordinate permutation

$$
(0)(1) \cdots(19)(20 \quad 23)(21 \quad 22) .
$$

\section{E. Constant Weight Lexicodes with a Sum Constraint;} $S(5,6,12)$

In view of Theorem 11 it is natural to ask if there is a similar definition for Mathieu's other famous Steiner system $S(5,6,12)$. The correct answer to this question emerged from some calculations of Ryba [18], which showed that this Steiner system can be obtained if a side condition is imposed on the lexicode.

A contant weight lexicode with sum $s$ is a constant weight lexicode as in Section IV-A, with the additional requirement that every codeword

$$
\cdots \zeta_{3} \zeta_{2} \zeta_{1} \zeta_{0}
$$

must satisfy

$$
\sum_{i} i \zeta_{i} \geq s
$$

where the sum is calculated as an ordinary integer. In other words (since the $\zeta_{i}$ are 0 or 1 ), the sum of the $w$ coordinates where the 1's are located must be at least $s$. Since every set of size $w$ sums to at least $\left(\begin{array}{l}w \\ 2\end{array}\right)$, the sum constraint is vacuous if $s \leq\left(\begin{array}{l}w \\ 2\end{array}\right)$. Then Ryba's discovery is the following.

Theorem 13 (Ryba [18]): The words of the constant weight lexicode with $w=6, d=4, n=12$, and sum constraint $s=21$ are the blocks (or hexads) of a Steiner system $S(5,6,12)$.

\footnotetext{
${ }^{3}$ Defined on $[16$, p. 59], for example.
} 
This may be easily verified by computer. Furthermore, the hexads are obtained with the so-called "shuffle labeling" described in [7, ch. 12].

Mathematical Blackjack (or Mathieu's Vingt-et-Un): The game for which this code (i.e., the hexads of $S(5,6,12)$ ) gives the winning positions may be called Mathematical Blackjack, or Mathieu's Vingt-et-un. Six cards from a deck of 12 cards labeled $\{0,1,2, \cdots, 11\}$ are laid out face upwards on the table. The two players move alternately, the move being to replace one of the laid out cards with any lower one chosen from the remainder of the deck. The first player to make the sum less than 21 loses. Then Theorem 13 is equivalent to the assertion that the winning strategy is always to move to a hexad from $S(5,6,12)$.

\section{F. Further Examples; New Lower Bounds to $A(n, 10, w)$}

The only example of a constant weight lexicode with a sum constraint for which we have a general theoretical result is the case $d=4, s=\left(\begin{array}{l}w \\ 2\end{array}\right)+1$. It can be shown that the codewords in this case are the winning positions in the misère version of Welter's game [1, pp. 480-481]. We omit the details.

When $d=10$, we discovered by experimenting that constant weight lexicodes with a sum constraint in many cases improved on the best previously known lower bounds for $A(n, 10, w)$ as given in [8, Table IV]. The results are shown in Table XIII. We also take the opportunity to correct an error in [8, Table IV]: the value of $A(16,10,7)$ should be 4 (not 3 ).

\section{TABLE XIII}

NEW LOWER BOUNDS ${ }^{a}$ ON $A(n, 10, w)$ OBTAINED FROM Constant Weight Lexicodes with SUm CONSTRainT $s$

$\begin{array}{ll}A(21,10,9) \geqslant 26 & \text { (use } s=59) \\ A(22,10,9) \geqslant 31 & \text { (use } s=70) \\ A(22,10,10) \geqslant 39 & \text { (use } s=92) \\ A(23,10,7) \geqslant 17 & \text { (use } s=58) \\ A(23,10,8) \geqslant 27 & \text { (use } s=54) \\ A(23,10,9) \geqslant 39 & \text { (use } s=74) \\ A(23,10,10) \geqslant 50 & \text { (use } s=75) \\ A(23,10,11) \geqslant 53 & \text { (use } s=80) \\ A(24,10,8) \geqslant 32 & \text { (use } s=54) \\ A(24,10,9) \geqslant 49 & \text { (use } s=65) \\ A(24,10,10) \geqslant 64 & \text { (use } s=82) \\ A(24,10,11) \geqslant 75 & \text { (use } s=79) \\ A(24,12,12) \geqslant 80 & \text { (use } s=96)\end{array}$

aAfter this work was completed, L. Hemachandra and V. K. Wei (personal communication) used the method of simulated annealing to show that $A(23,10,7) \geq 18, A(23,10,8) \geq 28$, and $A(24,10,8) \geq 33$.
Finally, we also experimented with applying a sum constraint to unrestricted lexicodes, but only found one code worth mentioning. When $B=2, d=3, n=8$ and $s=21$, the corresponding lexicode contains 18 codeswords. This is better than any linear code, although not as good as Julin's optimal code [13] with 20 words.

\section{ACKNOWLEDGMENT}

We thank A. I. F. Ryba for allowing us to include his result.

\section{REFERENCES}

[1] E. R. Berlekamp, J. H. Conway, and R. K. Guy, Winning Ways, 2 vols. New York: Academic, 1982.

[2] J. H. Conway, On Numbers and Games. New York: Academic, 1976.

[3] J. H. Conway and H. S. M. Coxeter, "Triangulated polygons and frieze patterns," Math. Gazette, vol. 57 pp. 87-94, 175-183, 1973.

[4] J. H. Conway, V. Pless, and N. J. A. Sloane, "Self-dual codes over GF (3) and GF (4) of length not exceeding 16," IEEE Trans. Inform. Theory, vol. IT-25, pp. 312-322, 1979.

[5] J. H. Conway and N. J. A. Sloane, "Laminated lattices," Ann. Math., vol. 116, pp. 593-620, 1982.

[6] - "Complex and integral laminated latices," Trans. Amer. Math. Soc., vol. 280, pp. 463-490, 1983.

[7] —, The Leech Lattice, Sphere Packings, and Related Topics. New York: Springer-Verlag, 1986, to appear.

[8] R. L. Graham and N. J. A. Sloane, "Lower bounds for constant weight codes," IEEE Trans. Inform. Theory, vol. IT-26, pp. 37-43, 1980.

[9] P. M. Grundy, "Mathematics and games," Eureka, vol. 2, pp. 6-8, 1939; reprinted in vol. 27, pp. 9-11, 1964.

[10] M. J. T. Guy, unpublished.

[11] R. K. Guy and C. A. B. Smith, "The G-values for various games," Proc. Camb. Math. Soc., vol. 52, pp. 514-526, 1956.

[12] A. A. Hashim and A. G. Constantinides, "Some new results on binary linear block codes," Electron. Lett., vol. 10, pp. 31-33, 19/4.

[13] D. Julin, "Two improved block codes," IEEE Trans. Inform. Theory, vol. IT-11, p. 459, 1965.

[14] H. W. Lenstra, Jr., On the algebraic closure of two," Konink. Nederl. Akad. Wetensch. Proc., vol. A80, pp. 389-396, 1977.

[15] - -, "Nim multiplication," in Séminaire de Théorie des Nombres 1977-1978, Centre National de Recherche Scientifique, Talence, France, Exp. 11, 1978.

[16] F. J. MacWilliams and N. J. A. Sloane, The Theory of Error-Correcting Codes, Amsterdam, The Netherlands: North Holland, 1977.

[17] P. Piret, "Good block codes derived from cyclic codes," Electron. Lett., vol. 10. pp. 391-392, 1974.

[18] A. J. E. Ryba, personal communication.

[19] R. P. Sprague, "Uber mathematische Kampfspiele," Tôhoku Math. $J .$, vol. 41, pp. 438-444, 1935-1936.

[20] C. P. Welter, "The advancing operation in a special abelian group," Koninkl. Nederl. Akad. Weten. Proc., vol. A55, pp. 304-314, 1952.

[21] _ - "The theory of a class of games on a sequence of squares, in terms of the advancing operation in a special group," Koninkl. Nederl. Akad. Weten. Proc., vol. A57, pp. 194-200, 1954.

[22] R. A. Wilson, "On lexicographic codes of minimal distance 4," Atti Sem. Mat. Fis. Univ. Modena, to appear. 